\title{
HYDROGEN PASSIVATION OF INTERSTITIAL Zn DEFECTS IN HETEROEPITAXIAL InP CELL STRUCTURES AND INFLUENCE ON DEVICE CHARACTERISTICS'
}

\author{
S.A. Ringel and B. Chatterjee \\ The Ohio State University \\ Department of Electrical Engineering \\ Columbus, $\mathrm{OH} 43210$
}

\begin{abstract}
Hydrogen passivation of heteroepitaxial InP solar cells is of recent interest for deactivation of dislocations and other defects caused by the cellvsubstrate lattice mismatch that currently limit the photovoltaic performance of these devices. In this paper we present strong evidence that, in addition to direct hydrogen-dislocation interactions, hydrogen forms complexes with the high concentration of interstitial $\mathrm{Zn}$ defects present within the $\mathrm{p}^{*}$ $\mathrm{Zn-doped} \mathrm{emitter} \mathrm{of} \mathrm{MOCVD-grown} \mathrm{heteroepitaxial} \mathrm{inP} \mathrm{devices,} \mathrm{resulting} \mathrm{in} \mathrm{a} \mathrm{dramatic} \mathrm{increase} \mathrm{of} \mathrm{the} \mathrm{forward}$ bias tum-on voltage by as much as $280 \mathrm{mV}$, from $\sim 680 \mathrm{mV}$ to $\sim 960 \mathrm{mV}$. This shitt is reproducible and thermally reversible and no such effect is observed for either n'p structures or homoepitaxial p'n structures grown under identical conditions. A combination of photoluminescence (PL), electrochemical C-V dopant profiling, SIMS and I$V$ measurements were performed on a set of samples having undergone a matrix of hydrogenation and posthydrogenation annealing conditions to investigate the source of this voltage enhancement and confirm the expected role of interstitial $\mathrm{Zn}$ and hydrogen. A precise correlation between all measurements is demonstrated which indicates that $Z n$ interstitials within the $\mathrm{p}^{*}$ emitter and their interaction with hydrogen are indeed responsible for this device behavior. Excess interstitial Zn defects are shown to introduce deep donor states detected by PL measurements that partially compensate the desired emitter acceptor doping and likely provide recombination centers that together act to reduce the device voltage. We find that hydrogenation completely deactivates these. donor states and that this passivation effect is thermally stable at $400^{\circ} \mathrm{C}$, the temperature at which $\mathrm{Zn}$ acceptors can be fully reactivated. This results in an increase in the emitter accoptor concentration by a factor of 2-3 after hydrogenation plus acceptor reactivation annealing since the compensating donor states remain deactivated. The thermal stability of this interstitial Zn passivation was found to be intermediate between $\mathrm{Zn}$ acceptor passivation and deep dislocation state passivation, partially reactivating after annealing at a temperature of 500 ${ }^{\circ} \mathrm{C}$ an completely reactivating above $550^{\circ} \mathrm{C}$, as determined by. $\mathrm{PL}$ measurements. We find that as the interstitial Zn donor state is reactivated by progressively higher temperature annealing, both the emitter doping and diode turn-on voltage systematically return to their original as-grown values, indicating the direct impact of interstitial $\mathrm{Zn}$ on device characteristics.
\end{abstract}

\section{INTRODUCTION}

Hydrogenation of heteroepitaxial InP solar cells grown on substrates such as $\mathrm{Si}$, Ge or GaAs is of recent interest as an approach to deactivate the electrical activity of dislocations within the InP device regions that arise from the large lattice mismatch between InP and the underlying substrate. [1,2] In previous work, we have extensively characterized this process and have shown that deep levels associated with dislocations are strongly passivated by hydrogen introduced in a post-growth plasma hydrogenation treatment.[3,4] Deep level

1 Work supported by NASA grant no. NAG3-1461 and by subcontract to Essential Research via NASA SBIR contract SBIR96-NAS327677-1 
concentrations were measured to decrease from near $1 \times 10^{15} \mathrm{~cm}^{-3}$ to the low $10^{12} \mathrm{~cm}^{-3}$ range after hydrogenation. This passivation was found to exhibit very strong thermal stability, with minimal deep level reactivation occurring up to anneal temperatures approaching $600 \mathrm{C}$, and was correlated with orders of magnitude suppression of reverse bias leakage currents of heteroepitaxial InP diodes. Similar deep level passivation and reverse current suppression characteristics were also observed for both $n^{\dagger} p$ and $p^{+} n$ cell structures.[5] However, in contrast to the reverse characteristics and deep level passivation, the forward bias I-V characteristics upon hydrogenation and annealing exhibited quite different behavior, which is summarized by dark I-V data in Figure 1. Comparison of the heteroepitaxial $p^{+} n$ and $n^{+} p$ structures (Figures $1 a$ and $1 b$ ) reveals a dramatic increase in the forward bias turn on voltage, $V_{\text {To, }}$ for the former with no such effect for the latter. Based on the fact that dislocations are similarly passivated for both structures as verified by Deep Level Transient Spectroscopy (DLTS) measurements, this voltage enhancement is unlikely to be directly related to dislocation passivation. Moreover, figure 1a shows that there are significant differences in the dependence of the forward and reverse bias characteristics on posthydrogenation annealing temperature even for the same p'n diode which indicates a different mechanism is controlling the forward current and reverse leakage characteristics. Taken together, these observations suggest that a process other than dislocation passivation is present within hydrogenated, heteroepitaxial p'n InP cell structures that also has a dramatic effect on device characteristics. It also implies that an additional and significant benefit can be realized by hydrogen passivation of heteroepitaxial p'n cells which are inherently limited by the performance of the p-type emitter.

The obvious difference in these cell structures is the presence of a high $\mathrm{Zn}$ concentration within the MOCVD-grown p-type emitter of the p'n configuration. Zn doping is a convenient but difficult dopant to control in MOCVD growth, particularly when ultra-sharp and high concentrations are needed, such as in a typical $p^{+}$emitter layer, due to the propensity for $\mathrm{Zn}$ to diffuse in InP. In addition, high $\mathrm{Zn}$ doping is known to lead to high concentrations of $\mathrm{Zn}$ atoms incorporated as interstitial defects $(\mathrm{Zn})$ which contribute deep donor bandgap states, rather than as desired substitutional defects which contribute shallow acceptor levels.[6] In an earlier paper, we have shown that the presence of dislocations within heteroepitaxial Zn-doped InP substantially increases the presence of interstitial $\mathrm{Zn}\left(\mathrm{Zn}_{1}\right)$ by a dislocation gettering mechanism.[7] Since interstitial $\mathrm{Zn}$ atoms introduce deep donors in the InP bandgap, we suspect that they may play a role in the observed dark I-V shift. Therefore, in this paper we explore this possibility by conducting a controlled set of experiments to elucidate the presence of interstitial $\mathrm{Zn}$ and its possible interaction with hydrogen, in order to explain the observed device I-V characteristics.

\section{EXPERIMENTAL}

InP solar cell structures having a p'n configuration were grown by MOCVD on both GaAs and InP substrates to directly compare the effects of hydrogen and interstitial Zn defects with and without the presence of dislocations. A schematic of the device structures is shown in Figure 2. The target emitter and base doping concentrations in all cases are $2 \times 10^{16} \mathrm{~cm}^{4}$ and $1 \times 10^{17} \mathrm{~cm}^{-3}$, respectively, with an emitter thickness of 0.15 um. All structures were grown by low pressure metalorganic chemical vapor deposition (MOCVD) using trimethyl In and phosphine for InP growth with silane and diethyl Zn providing n-type (Si) and p-type (Zn) doping, respectively. Complete details of the growth procedure have been reported elsewhere.[8] For the p'n structures, identical Zn and Si doping proffles were targeted for all heteroepitaxial and homoepitaxial structures so that the latter can provide a "control" sample set for comparison. Additional $n+p$ cell structures were also investigated for additiona! confirmation of the results.

Post-growth hydrogenation was performed using a $13.56 \mathrm{MHz}$ parallel plate plasma reactor. Immediately prior to hydrogenation, a thin, $\mathrm{H}$-permeable protective $\mathrm{SiN}_{\mathrm{x}}$ coating was deposited onto the InP surface to avoid preferential loss of phosphorous and surface degradation due to sustained exposure to the H-plasma.

Hydrogenation was performed using a substrate temperature of $250^{\circ} \mathrm{C}$ at a plasma power density of $0.08 \mathrm{~W} / \mathrm{cm}^{2}$ for 2 hours at a total pressure of $750 \mathrm{mTorr}$. After plasma hydrogenation, acceptor and donor dopant atoms were reactivated by a 5 minute $400^{\circ} \mathrm{C}$ anneal in flowing nitrogen followed by chemical removal of the protective silicon nitride cap. We have previously shown this to result in extensive passivation of deep levels associated with dislocations and other extended defects throughout the entire depth of the InP devices coupled with complete reactivation of the desired dopants. Details of the process have been described in earlier publications. [2-4] For samples selected for thermal stability studies after the dopant reactivation step, additional heat treatments for 5 
minutes in flowing nitrogen at either $500^{\circ} \mathrm{C}$ or $600^{\circ} \mathrm{C}$, depending on the particular experiment, were performed prior to nitride cap removal. For device testing, mesas were etched to define device areas and ohmic contacts were formed to the p'n junctions by electron beam evaporation using $\mathrm{Ag}-\mathrm{Zn}$ and AuGeNi for p-type and $n$-type InP surfaces, respectively. Samples were characterized using a combination of electrochemical C-V dopant profiling, photoluminescence (PL), dark I-V and SIMS measurements at all stages of the hydrogenation and annealing process to monitor changes within the material.

\section{RESULTS AND DISCUSSION}

\subsection{Comparison of Dark I-V Characteristics}

As mentioned above, Figure 1 depicts a representative set of the I-V characteristics observed for heteroepitaxial p'n and $n$ 'p InP cell structures, along with homoepitaxial InP/InP $p+n$ device to provide a comparison for the p'n structure without the presence of dislocations. After receiving the hydrogenation plus 400 ${ }^{\circ} \mathrm{C}$ dopant reactivation anneal, the reverse characteristics of both heteroepitaxial configurations displayed similar improvement in the reverse leakage current, and subsequent higher temperature annealing demonstrated a similar reactivation behavior. The temperatures at which the reverse leakage current reactivation are observed track the deep level reactivation associated with the dissociation of hydrogen-dislocation deep state complexes previously measured by DLTS.[4] In fact, results of Arrhenius analysis of the reverse current activation as a function of temperature were also previously found to match the dominant deep state in each case, indicating the correlation between dislocation-related deep states and reverse leakage current.

However, the forward I-V behavior between the two configurations deviate considerably. The optimum 2. hour hydrogen exposure plus $400^{\circ} \mathrm{C}$ dopant reactivation anneal increases the forward bias tum on voltage $\left(V_{\text {ro }}\right)$ by $280 \mathrm{mV}$ for the p'n structure, from $680 \mathrm{mV}$ to $960 \mathrm{mV}$. We have also found that the magnitude of this shift is proportional to the duration of the hydrogen exposure, with saturation occurring beyond 2 hours. No such effect is observed for the n'p heteroepitaxial case and a negligibly small increase is observed for the homoepitaxial p'n structure. It is useful to note that the fonwand I-V characteristics for the homoepitaxial and heteroepitaxial p'n structures after hydrogenation are now very similar in terms of $V_{\text {ro }}$. This important similarity will be discussed later. Additional inspection of figure 1a reveals that, even for the same p'n diode, there are clear differences in the reactivation of the reverse and forward bias characteristics. Both the reverse leakage current and forward injection/diffusion current characteristics are markedly improved by the hydrogenation plus $400^{\circ} \mathrm{C}$ dopant reactivation process, with current reduction in the former and a positive voltage shift in the latter. After a $500^{\circ} \mathrm{C}$ anneal, the reverse current magnitude is retained at the passivated value, while the forward I-V has begun to shift back toward its original characteristic prior to passivation. Incremental annealing at higher temperatures results in a progressive recovery to the original forward I-V behavior. No such annealing dependence is observed for the reverse bias characteristics, which instead are quite stable up to temperatures in excess of $550^{\circ} \mathrm{C}$. This observation, coupled with our earlier results which correlated the reverse leakage current directly to dislocation-related current generation, suggests a different process is likely to be responsible for the observed dependence of the forward bias characteristics on post hydrogenation annealing.

\subsection{Effects of Hydrogenation and Annealing on Emitter Doping and Zn Distribution}

A series of experiments were performed to investigate whether $\mathrm{Zn}$ was involved in the observed I-V behavior. $\mathrm{Zn}$ is a likely suspect for device instabilities due to its high diffusion coefficient and its propensity to occupy interstitial sites within InP. Figure 3 shows the results of electrochemical C-V dopant profiling of p'n heteroepitaxial and homoepitaxial structures that have undergone the hydrogen processes described in the caption. The as-grown emitter doping concentration for the heteroepitaxial sample is slightly lower than its homoepitaxial counterpart for identical DEZn flow conditions, which we have consistently observed when comparing identically grown homoepitaxial and heteroepitaxial $\mathrm{Zn}$ doped InP layers. In an earlier paper we showed this to result from a higher concentration of $Z n_{1}$ defects in the heteroepitaxial layer which will lower the hole concentration by competing with the desired incorporation of $\mathrm{Zn}$ as substitutional acceptors and by compensating the p-type material through the introduction of deep donor bandgap states.[7] Immediately after hydrogenation and prior to any reactivation anneal, the emitter acceptor concentration reduces by more than two

orders of magnitude for both cases, as expected. The donors within the base are not passivated by hydrogen as seen by the unchanged dopant concentration in the base of both structures. A subsequent $400^{\circ} \mathrm{C}$ anneal is 
expected to reactivate the shallow acceptors to recover their as-grown concentration, and this does occur for the homoepitaxial sample. However, we consistently find that the net acceptor concentration within the heteroepitaxial devices are actually reactivated to values that are a factor of 2-3 larger than their as-grown concentration. This is clearly observed in figure 3. In fact, this concentration now closely matches that of the homoepitaxial sample. Further annealing at progressively higher temperatures results in a systematic decrease in the emitter doping concentration, eventually reaching the as-grown value after a 5 minute anneal at temperatures in excess of $550^{\circ} \mathrm{C}$.

These observations closely track the evolution of the dark I-V characteristics as a function of hydrogenation and annealing. To investigate whether a redistribution of the $\mathrm{Zn}$ profile can explain these observations, SIMS measurements were performed on the same sample matrix. The results are summarized by the series of SIMS profiles in figure 4. Only the $\mathrm{Zn}$ and $\mathrm{H}$ profiles were plotted here, although In, P, Si, C and O were also profiled. From this figure, it is clear that the $\mathrm{Zn}$ dopant profile is not significantly affected by either the hydrogenation process or the subsequent annealing at the temperatures used here. Quantitative variations in the average Zn concentration within the emitter are within a few percent over this sample matrix, whereas the acceptor concentration as measured by CV varied by a factor of $\sim 2$. A similar disparity is also evident by comparing the as-grown heteroepitaxial and homoepitaxial samples where a factor of $\sim 2$ change is observed for CV acceptor concentration but SIMS indicates a comparable total $\mathrm{Zn}$ dopant concentration. At this time, it is instructive to note that the hydrogen profile closely tracks the $\mathrm{Zn}$ profile, implying a possible connection between the two that will become evident later. In the next section, we provide evidence that the source of this discrepancy between acceptor concentration and total $\mathrm{Zn}$ doping is the presence of a high concentration of interstitial $\mathrm{Zn}\left(\mathrm{Zn}_{\mathbf{n}}\right)$ defects.

\subsection{Effect of Passivation on Photoluminescence Spectra and Evidence for Passivation of Interstitial Zn}

Figures 5 and 6 show low temperature PL spectra measured on homoepitaxial and heteroepitaxial samples processed as described above. All PL measurements were performed under the same conditions (temperature, incident laser intensity, etc.) so that meaningful comparisons can be made. A brief description of the features within the PL spectra is necessary prior to discussing connections to the earlier observations. The conduction band to acceptor (C-A) peak (and its phonon replica) is a signature of -type inP and typically dominates p-InP PL spectra.[6,7] For n-InP however, the dominant feature is a band to band (B-B) peak with no C-A peak expected. This has been confirmed by performing PL measurements on individual epilayers. Since here PL is performed directly on the device structures, the PL linescans shown in figures 5 and 6 are actually composites of the PL emission from the 0.15 um thick $\mathrm{p}^{+}$emitter and the underlying $n$-type base region, although the emitter is expected to be the dominant contributor due to the strong absorption of the $488 \mathrm{~nm}$ PL excitation source within the surface region of the InP samples.

We first discuss differences in the as-grown PL spectrum of each figure, which leads to two important, initial observations. First, the overall magnitude of the PL spectra for the homoepitaxial as-grown sample in figure 5 is about a factor of 40 larger in magnitude than the PL spectra for the heteroepitaxial as-grown sample in figure 6. This is expected as the overall material quality of the homoepitaxial samples are superior to that of the heteroepitaxial samples. Second, while the overall PL spectrum is reduced for the heteroepitaxial sample, the quality of the emitter and base layers, as characterized by the B-B and C-A peak intensities respectively, appear to have degraded differently upon direct comparison between the as-grown spectra. The C-A peak, which is a signature of the PL emission from the Zn-doped emitter, is much larger than the B-B peak due to PL emission from the n-type base for the homoepitaxial sample, indicating that the majority of the PL response is from the emitter layer, as expected based on absorption of the excitation laser light. In contrast, for the as-grown heteroepitaxial PL spectrum in figure 6, the C-A peak is actually suppressed relative to the B-B peak, suggesting that a larger fraction of the overall PL emission is emanating from the $n$-type base. The reason for this is clear upon further inspection of figures 5 and 6 where a broad PL emission centered near 1.25 eV is also evident. This feature is easily detected in the heteroepitaxial as-grown sample and results from a donor-acceptor (D-A) transition between $\mathrm{Zn}$, donor-like levels and $\mathrm{Zn}$ substitutional acceptor levels. This $\mathrm{D}-\mathrm{A}$ peak is generally observed for $\mathrm{Zn}$ doping concentrations in excess of mid $10^{17} \mathrm{~cm}^{-3}$ range for InP. In some earlier work, we have shown that the D-A intensity is greatly enhanced for heteroepitaxial samples as compared to identically doped homoepitaxial samples that was explained as a $Z n$, gettering phenomena due to dislocation strain fields, which ultimately increases the $\mathrm{Zn}$, concentration at the expense of substitional $\mathrm{Zn}$. [7] This difference is clear from figures 5 and 6 where the D-A peak for the homoepitaxial sample is evident only in the magnified inset in figure 5. 
From these PL studies, the $\mathrm{Zn}$, concentration as indicated by $\mathrm{D}-\mathrm{A}$ peak intensities was estimated to be as much as $50 \%$ of the total $\mathrm{Zn}$ concentration for $\mathrm{Zn}$ doping in the $10^{18} \mathrm{~cm}^{-3}$ concentration range. Therefore, a large concentration of $\mathrm{Zn}_{1}$ defects within the heteroepitaxial $\mathrm{p}^{+}$emitter here is certainly expected. Therefore, due to the presence of both dislocations and $\mathrm{Zn}$ dopants, the heteroepitaxial $\mathrm{p}^{+}$emitter undergoes a severe degradation that is not shared by the $n$-type base, hence the observed suppression of the C-A peak in the as-grown heteroepitaxial PL spectrum.

Now that the $Z n$, defect has been discussed via the observation of the D-A PL peak, and the general features of the PL spectra have been described, the effects of hydrogenation and post-hydrogenation annealing on the PL characteristics can be considered. After the 2 hour hydrogen exposure and the $400^{\circ} \mathrm{C}$ dopant reactivation anneal, the C-A and B-B peaks increase in intensity as seen in figures 5 and 6 , whereas the D-A peak is completely suppressed by hydrogen incorporation. Since the concentration of shallow $\mathrm{Zn}$ acceptors have been fully reactivated according the CV data shown earlier, the D-A peak suppression can only be due to the deactivation (effective elimination) of the $\mathrm{Zn}$, donor states. To our knowledge; this is the first direct evidence of $\mathrm{Zn}$, passivation in any III-V semiconductor, and indicates a different passivation mechanism for $\mathrm{Zn}$ in its interstitial form versus $\mathrm{Zn}$ in its substitutional acceptor form since the latter is fully reactivated by the same $400^{\circ} \mathrm{C}$ anneal. A detailed and quantitative study of the $\mathrm{Zn}$, passivation process and properties in both heteroepitaxial and homoepitaxial InP is the subject of a publication in progress.

PL measurements were performed after subsequent higher temperature anneals in order to compare with the doping and I-V behavior already discussed. Both figures 5 and 6 show that the D-A peak is partially reactivated by a $500{ }^{\circ} \mathrm{C}$ anneal, and is fully reactivated after a $600{ }^{\circ} \mathrm{C}$ anneal for both heteroepitaxial and homoepitaxial device structures. This evolution of the PL spectra precisely tracks both the evolution in the $\mathrm{Zn}$ acceptor concentration shown in figure 3 and the forward bias I-V characteristics shown in figure 1, providing a remarkable correlation between the D-A PL peak behavior, the emitter doping behavior and the device forward I$V$ characteristics. This is strong evidence for the direct involvement of interstitial $\mathrm{Zn}$ in the heteroepitaxial $p^{*} n$ device behavior.

\subsection{Comparison of 1-V. Dopant Profiling and Photoluminescence results: Impact of $\mathrm{H}-\mathrm{Zn}$ Interactions}

Taken together, the data described above leads to the following model. The high concentration of $\mathrm{Zn}$, defects within the heteroepitaxial $\mathrm{Zn}$-doped emitter is passivated by hydrogen, along with $\mathrm{Zn}$ acceptors as well as deep dislocation states. A $400{ }^{\circ} \mathrm{C}$ reactivates only the $\mathrm{Zn}$ acceptors, leaving dislocation states and the deep donor states associated with interstitial Zn passivated. The deactivation of the deep donors eliminates their ability to compensate the shallow $\mathrm{Zn}$ acceptor levels, effectively increasing the electrically active $\mathrm{Zn}$ acceptor concentration. This is confirmed by the SIMS data which showed no change in the total Zn concentration, which is the sum of the substitutional acceptor and interstitial donor concentrations. At higher annealing temperatures, the $\mathrm{Zn}$, donors become reactivated as the $\mathrm{H}-\mathrm{Zn}$, complex (the exact form of which is currently under investigation) dissociates. This then begins to re-compensate the $\mathrm{Zn}$ acceptors, ultimately reducing the emitter doping concentration back to the original as-grown value. Hence, the PL, SIMS and CV data are consistent with each other and the variation of acceptor doping is completely explained by the passivation and reactivation of $\mathrm{Zn}$, donor-like defect states.

The trend in passivation and thermal reactivation of $\mathrm{Zn}$, defects is also consistent with the forward I-V data of figure 1. An increase in the emitter doping after hydrogenation plus the $400{ }^{\circ} \mathrm{C}$ dopant reactivation anneal due to reduced compensation will translate into an increase in the fundamental built in voltage of the pn junction, consistent with the increase in the turn on voltage, $V_{\text {ro, }}$ shown by the $\mathrm{H}-\mathrm{V}$ data of the heteroepitaxial p'n device in figure 1a. However, some simple calculations show the increase in the ideal built in voltage expected for this factor of 2-3 increase in emitter doping is only $30 \mathrm{mV}$, not nearly enough to explain the observed $280 \mathrm{mV}$ increase. Another possibility is a reduction in recombination current and/or increase in shunt resistance. This is highly plausible since the measured value of $\mathrm{V}_{\mathrm{TO}}$ is far less than the theoretical built in voltage for InP and voltage loss through a parallel path can be expected, especially for highly defective material having low carrier lifetimes and since the likelihood of excess interstitial $\mathrm{Zn}$ incorporated within the depletion region is high. Whether the source of the recombination is related to interstitial $\mathrm{Zn}$ defects, and if hydrogen effectively suppresses this recombination current is currently under investigation. Nevertheless, it is clear from these results that $\mathrm{Zn}$, and $\mathrm{H}$ $\mathrm{Zn}$, interactions have a direct impact on heteroepitaxial p'n InP device characteristics, and given the temperature range at which these complexes dissociate, such interactions may also be an issue for typical MOCVD growth of InP where $\mathrm{Zn}$ is used as a dopant. 


\section{Conclusions}

In this paper we have shown that a high concentration of interstitial $\mathrm{Zn}$ defects are present within the emitter of $p^{*} n$ heteroepitaxial InP solar cell structures, and have demonstrated that they are efficiently passivated by post-growth hydrogenation. Interstitial $\mathrm{Zn}$ defects were shown to be responsible for the low turn-on voltages characteristic of heteroepitaxial p'n InP diodes, and were also shown to compensate the desired maximum emitter doping concentration. Post-growth hydrogenation was found to efficiently deactivate the deep donors associated with the interstitial Zn defects, displaying a thermal stability in excess of the common Zn acceptor passivation. Thus, the beneficial effects of the $\mathrm{Zn}$, passivation can be realized. We find this to be directly responsible for a factor of $2-3$ increase in the maximum acceptor doping achievable in the heteroepitaxial $\mathrm{p}^{*}$ emitter, as well as a remarkable increase in the device voltage output, with an increase from $680 \mathrm{mV}$ to $960 \mathrm{mV}$ being observed for the forward bias tum-on dark voltage. This is an important technological result if it can be sustained in an actual operating solar cell (currently under investigation) since the emitter and $\mathbf{V}_{\infty}$ in general limit the performance of heteroepitaxial p`n InP cells.

\section{References}

1. R. K. Jain and D. J. Flood, "Influence of the Dislocation Density on the Performance of Heteroepitaxial InP Solar Cells", IEEE Trans Elec. Devices, vol. 40, pp. 128-134, 1993.

2. S.A. Ringel, "Hydrogen-Extended Defect Interactions in Heteroepitaxial InP Materials and Devices," Sol. State Electron. 41, pp. 359-380, 1997.

3. B. Chatterjee and S. A. Ringel, "Hydrogen Passivation and its Effects on Carrier Trapping by Dislocation is InP/GaAs Heterostructures", J. Appl. Phys. vol. 77, pp. 3885-3898, 1995.

4. B. Chatterjee, S. A. Ringel, R. Sieg, R. Hoffman, and I. Weinberg, "Hydrogen Passivation of Dislocation in InP on GaAs Heterostructures", Appl. Phys. Lett. vol. 65, pp. 58-61, 1994.

5. B. Chatterjee, S. A. Ringel, and R. Hoffman, "Hydrogen Passivation of n'p and p'n Heteroepitaxial InP Solar Cell Structures", Progress in Photovoltaics, vol. 4, pp. 91-100, 1996.

6. G. J. Van Gurp, T. Van Dongen, G. M. Fontijn, J. M. Jacobs, and D. L. A. Tjaden, "Interstitial and Substitutional $Z n$ in InP. and InGaAsP", J. Appl. Phys. vol. 65, pp. 553-560, 1988

7. R. M. Sieg, B. Chatterjee, and S. A. Ringel, "Evidence for Enhanced Zn Interstitial Concentration in StrainRelaxed Heteroepitaxial InP", Appl. Phys. Lett. vol. 66, pp. 3108-3110, 1995.

8. R. W. Hoffman, N. S. Fatemi, D. M. Wilt, P. Jenkins, D. J. Brinker, and D. A. Scheiman, "High Efficiency InP

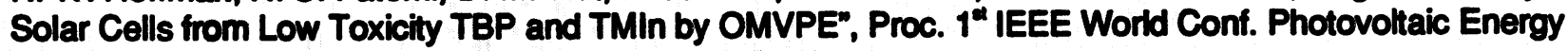
Conv. Pp. 1882-1885, 1994. 

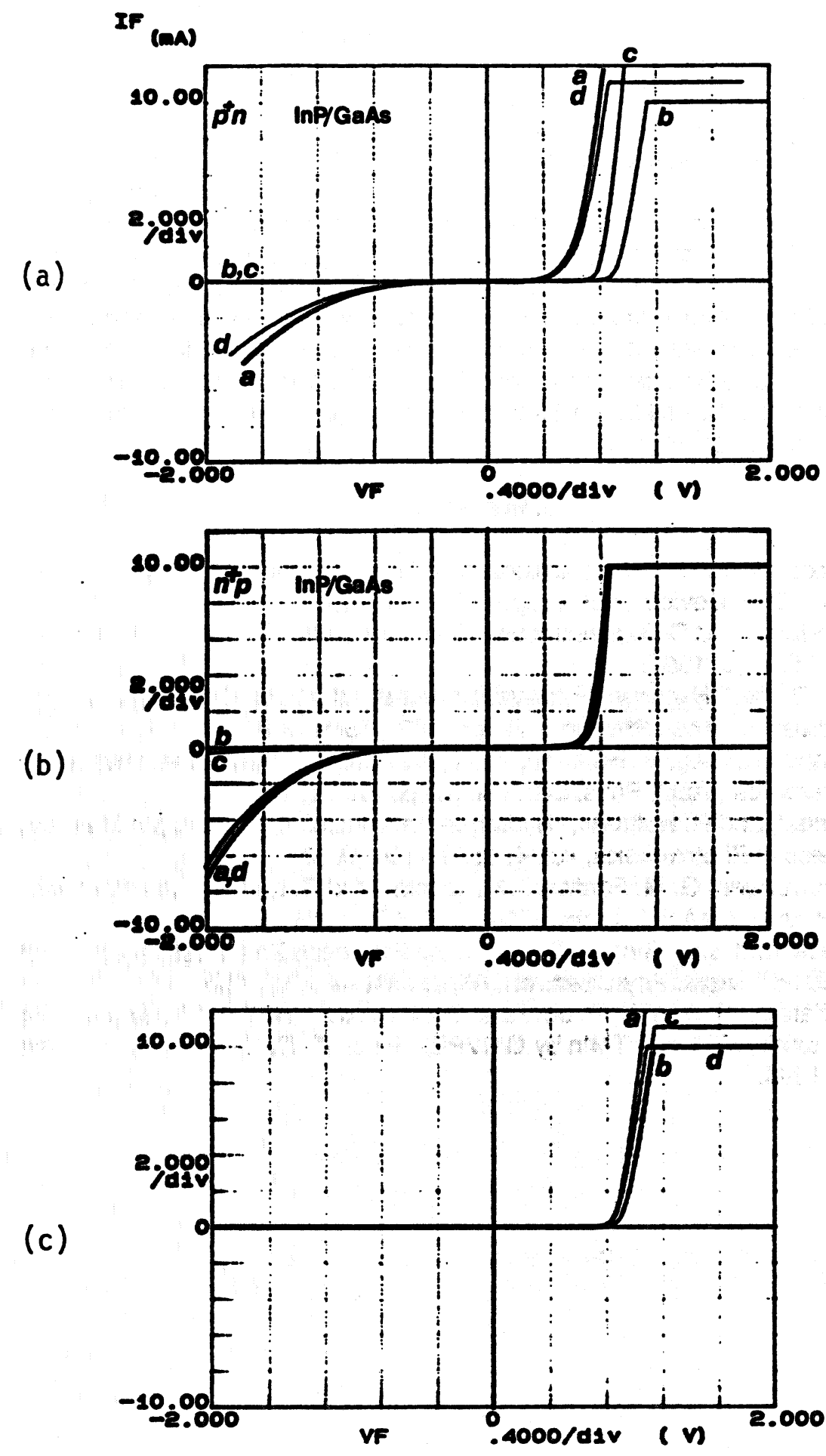

Figure 1. Dark I-V characteristics of (a) heteroepitaxial $p^{+} n$, (b)heteroepitaxial $n^{+} p$ and (c) homoepitaxial $p^{+} n$ InP cell structures. In each case, the labels a-d correspond to the following conditions; as-grown, hydrogenation $+400^{\circ} \mathrm{C}$ dopant reactivation anneal, hydrogenation $+500^{\circ} \mathrm{C}$ anneal and hydrogenation $+600^{\circ} \mathrm{C}$ anneal, respectively. The magnitude of the forward bias shift in figure $1(a)$ is $280 \mathrm{mV}$. 


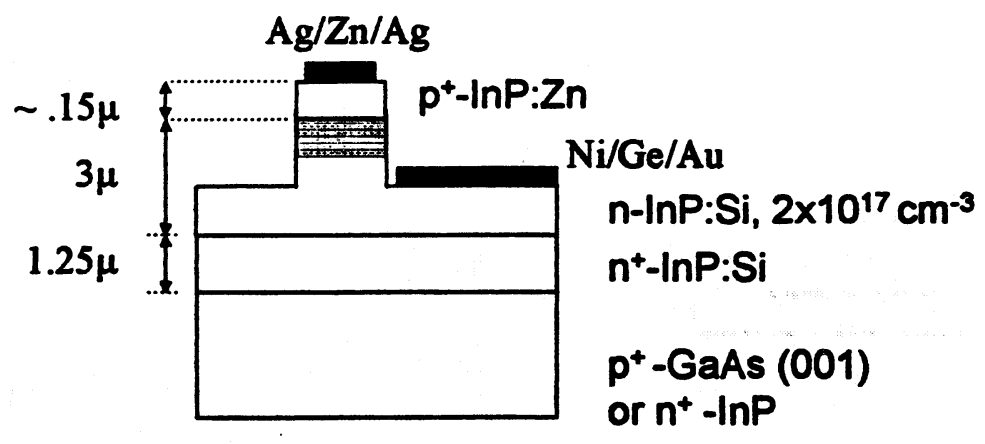

Figure 2. Schematic of the $p^{+} n$ device structures used in this study.
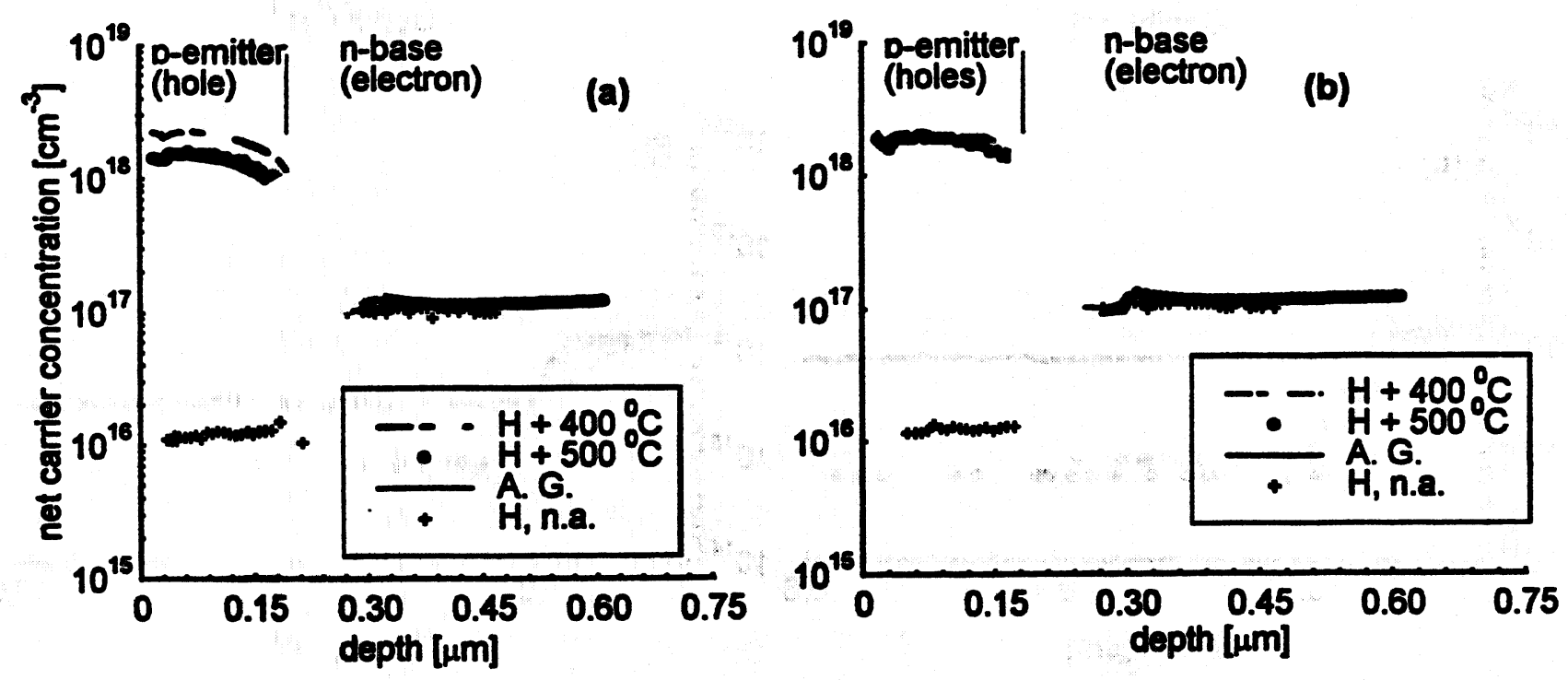

Figure 3. Electrochemical C-V dopant profiles measured for (a) heteroepitaxial and (b) homoepitaxial $p^{+} n \ln P$ structures for as-grown, after hydrogenation with no anneal, after hydrogenation $+400^{\circ} \mathrm{C}$ dopant reactivation anneal and after hydrogenation $+500{ }^{\circ} \mathrm{C}$ anneal, as indicated. The increase in emitter doping concentration is clearly observed for figure 3 a after the optimum passivation process. Note that the donors in the n-type base are not passivated by hydrogen, as' expected. 

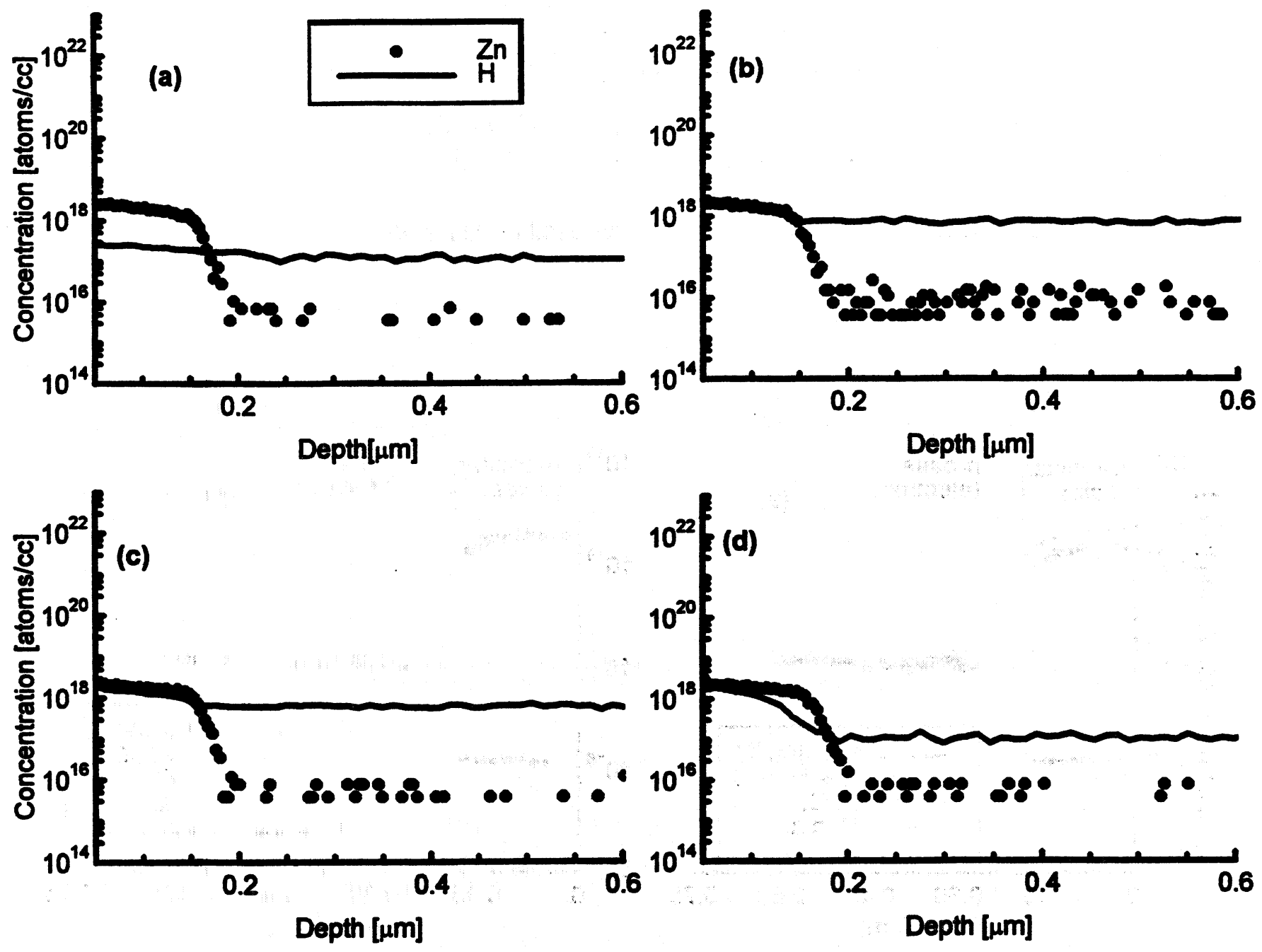

Figure 4. SIMS profiles for the heteroepitaxial $p^{+} n$ structures indicating the distribution of $\mathrm{Zn}$ and $\mathrm{H}$ for (a) as-grown samples, (b) after hydrogenation $+400^{\circ} \mathrm{C}$ annealing, (c) after hydrogenation $+500^{\circ} \mathrm{C}$ annealing, and (d) after hydrogenation $+600^{\circ} \mathrm{C}$ annealing. The evolution of hydrogen is evident for case (d): 